Editorial

\title{
Chess endgame table base records
}

\section{Introduction}

Before the construction of seven-piece bases the longest known win in the endings was a win in 243 moves (before conversion) in the ending "a rook and a knight against two knights", found by Lewis Stiller in 1991. Later it has been established that the record to mate in this class of the endings makes 262 moves. It has appeared that in the seven-piece endings there are longer wins. A win in 290 moves in the ending "two rooks and a knight against two rooks" became the first of them. Further variety of records has been found still. In May 2006 the position with a win in 517 moves has been found in the ending a queen and a knight against a rook, a bishop and a knight. Black to move and white wins in 517 moves (Figure 1). In general in many pawn less endings more than 50 moves before conversion are necessary for a win, and it was already known and for a number of six-pieced and even the five-pieced endings, even such ending, as "a queen and a rook against a queen". Even in pawn full endings there are many positions where a huge number of moves are needed for win. White wins in 260 moves (Figure 2). In this situation the rule of 50 moves, in Yakov Konoval's opinion, demands specification, differently it breaks logic of chess struggle. In a chess composition this rule it is not taken at all into consideration, besides it is easy to find positions where this rule is taken in account, but in absolutely drawn positions it is possible to play more than 500 moves. More than 500 moves can be played (Figure 3).

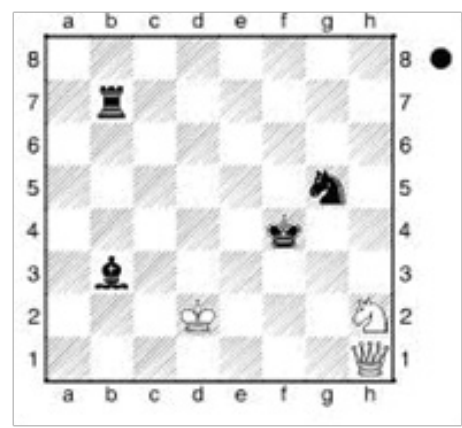

Figure I Black to move and white wins in 517 moves.

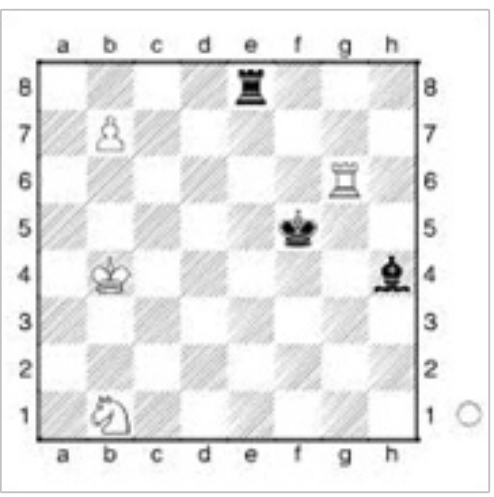

Figure 2 White wins in 260 moves.
Volume 3 Issue 5 - 2019

\author{
Yakov Konoval, Karsten Müller \\ Designation Computer Chess, University Orenburg State \\ University, Russia
}

Correspondence: Karsten Müller, Designation Computer Chess, University Orenburg State University, Russia, Tel 7-3532-777362,Email yashak@mail.ru

Received: November 20, 2017 | Published: December 06, 2017

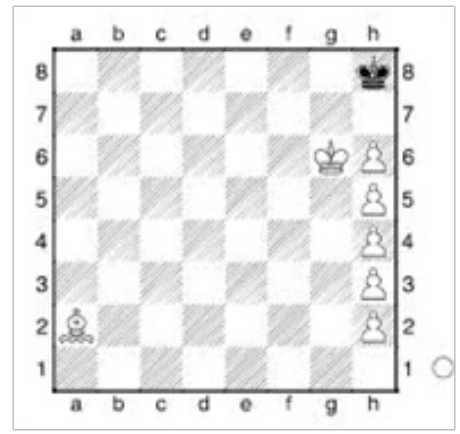

Figure 3 More than 500 moves can be played.

Karsten Müller thinks that for a human over the board play the 50 move shall remain as human play is not perfect anyway. There are two interesting results achieved by Marc Bourzutschky and Yakov Konoval already in 2009. The first of them - construction of several eight-piece bases without pawns in which one of the sides has two dark-squared and two light-squared bishops. The most interesting of them is the base "four bishops against two rooks". It has appeared that generally four bishops win, and it is necessary more than 50 moves for a win. The second result is the usage of the idea about the limited promotions for finding of positions in which two or even three under promotions into different pieces are necessary for a win. This approach was suggested by well known mathematician and chess composer Noam Elkies and was implemented by Marc and Yakov. Thus the computer acts in a role of the composer of chess studies, the man needs only to analyze the found positions and to decide, whether is they ready studies or not, at desire it is possible to add additional moves. In fact, 2 studies by computer were already published and according to experts in chess composition (Oleg Pervakov and others) they are good enough. White wins (Figures 4) (Figures 5). These studies were composed by computer. In 2012 the team from the Moscow State University (Vladimir Makhnychev and Victor Zakharov) constructed 7-man EGTBs using the supercomputer Lomonosov in the DTM format. Now their EGTBs are available online (for some payment) and it is possible to verify online any 7-man position (except 6 pieces versus lonely king). 


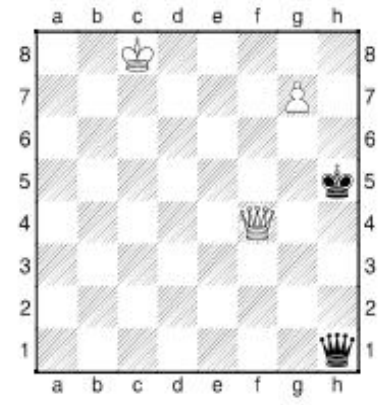

Figure 4White wins.

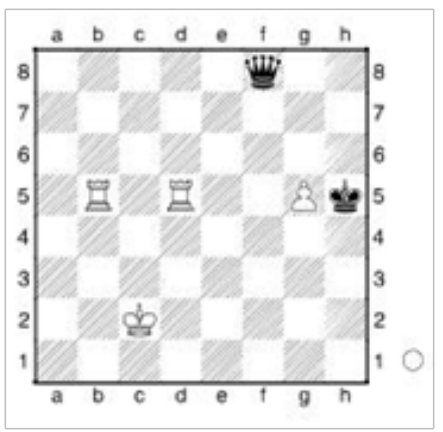

Figure 5 White wins.

\section{Some main events in the history of creating chess endgame table bases:}

a. In 1965, Richard Bellman proposed the creation of a database to solve chess and checkers endgames using retrograde analysis. Instead of analyzing forward from the position currently on the board, the database would analyze backward from positions where one player was checkmated or stalemated. Thus, a chess computer would no longer need to analyze endgame positions during the game because they were solved beforehand. It would no longer make mistakes because the table base always played the best possible move.

b. In 1970, Thomas Strohlein published a doctoral thesis with analysis of the following classes of endgame: KQK, KRK, KPK, KQKR, KRKB, and KRKN.

c. In 1977 Thompson's KQKR database was used in a match versus Grandmaster Walter Browne.

Ken Thompson and others helped extend table bases to cover all four- and five-piece endgames, including in particular KBBKN, KQPKQ, and KRPKR. Lewis Stiller published a thesis with research on some six-piece table base endgames in 1995. More recent contributors have included the following people:

a. Eugene Nalimov, after whom the popular Nalimov table bases are named b. Eiko Bleicher, who has adapted the table base concept to a program called "Freezer"' Guy Haworth, an academic at the University of Reading, who has published extensively in the ICGA Journal and elsewhere

Marc Bourzutschky and Yakov Konoval, who have collaborated to analyze endgames with seven pieces on the board; Peter Karrer, who constructed a specialized seven-piece table base (KQPPKQP) for the endgame of the Kasparov versus The World online match; Vladimir Makhnychev and Victor Zakharov from Moscow State University, who completed 4+3 DTM-table bases (525 endings including KPPPKPP) in July 2012. The table bases are named Lomonosov table bases. The next set of 5+2 DTM-table bases (350 endings including KPPPPKP) was completed during August 2012. The high speed of generating the table bases was because of using a supercomputer named Lomonosov. The size of all table bases up to seven-man is about $140 \mathrm{~TB}$. The table bases of all endgames with up to six pieces are available for free download, and may also be queried using web interfaces. Nalimov tablebases up to 6 men requires more than one terabyte of storage space.

Regarding human understanding John Nunn's outstanding 3 works Secrets of Rook Endings, Secrets of Minor Piece Endings and Secrets of Pawn less Endings are milestones regarding the 5 men endings. ${ }^{1-3}$ Nunn's Chess Endings 1 and 2 (GAMBIT 2010) reveal a lot about 6 men endings and Understanding Rook Endings by Müller \& Konoval ${ }^{4,5}$ (GAMBIT 2016) opened the door to 7 men rook endings. But the process of human understanding of the table base results has just begun and we are curious, what the future will bring and how much light can be shed on this deep and dark ocean. There are some new approaches in Konoval's table base generator, which allow generating 7-man table bases pretty fast. In fact, it was the first generator for 7-man chess table bases. Moreover, it works on usual home computer. Later, in 2012, 7-man table bases were generated by Lomonosov team ${ }^{6}$ with usage of very powerful supercomputer.

\section{Acknowledgments}

No acknowledgement.

\section{Conflict of interest}

No conflict of interest.

\section{References}

1. Nunn John. Secrets of Rook Endings. 2nd ed. GAMBIT. 1999. p. 1-352.

2. Nunn John. Secrets of Pawn less Endings. 1994

3. Nunn John. Secrets of Minor Piece Endings. 1995.

4. Muller K, Konoval Y. Understanding Rook Endgames. Gambit. 2016. p. $1-288$.

5. Muller K, Konoval Y. Understanding Minor Piece Endings. 2017.

6. Lomonosov team. 7-man depth-to-mate table base service. 2017 